\title{
Pre-Operative Evaluation of an Orbital Injury
}

\author{
Valerio Massimo Magro $^{1^{*}}$ and Maria Cristina Magnotti ${ }^{2}$ \\ ${ }^{1}$ Department of Internal Medicine and Geriatric, University of Campania "Luigi Vanvitelli", Italy \\ ${ }^{2}$ Department of Odontostomatological and Maxillo-Facial Sciences, Policlinico Umberto 1, Italy
}

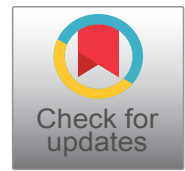

*Corresponding author: Dr. Valerio Massimo Magro, MD, Department of Internal Medicine and Geriatric, University of Campania "Luigi Vanvitelli", Piazza Miraglia 2, 80138, Naples, Italy, Tel: 3492224922, E-mail: valerio_magro@hotmail.com

\begin{abstract}
Intraocular foreign bodies are a major cause of ocular trauma and blindness. Young adults, especially men, are the most likely victims, as a result of industrial or agricultural injuries. The management of orbital foreign bodies remains a serious diagnostic problem, despite development of diagnostic imaging techniques. Ophthalmic examination, including visual acuity assessment, is necessary before and after surgical intervention. With computed tomography accurate foreign body localization is possible.
\end{abstract}

\section{Keywords}

Eye, Orbital injury, Imaging, Critical care medicine, Maxillofacial Surgery

\section{Introduction}

Penetrated injuries are most difficult injuries of the eye. Intraocular Foreign Body (IOFB) may lodge in any of the structures it encounters, from anterior chamber to the retina and choroid. The management of IOFB remains a serious diagnostic problem, despite development of diagnostic imaging techniques. Here we present a case came to the observation to describe the outlines of our supervision.

\section{Case Report}

A 35-year-old man suffered an injury to his right eye. He presented to an emergency department with the presence of a penetrating injury and an intraocular metallic foreign body. The patient was working as a lawnmower and employing a mechanical device to cut the lawn had hooked a piece of iron remained hidden in the tall grass. At a first clinical examination the clinical condition of the subject appeared discrete. Patient intenseIy anxious. We proceeded to a clinical and instrumental evaluation (fundus oculi) and with imaging (computed tomography, CT) (Figure 1: Panel A, Panel B). We found good eye pressure at the digital pressure. At the intra- operative examination, the chamber was formed and round and center-shaped optical papilla. Fortunately there was a minimal involvement of intraocular structures with no significative retinal damage. Visual acuity was difficult to evaluate pre-operably but did not seem to be greatly reduced or there were signs of retinal detachment due to massive inflammatory edema. We appreciated a Berlin's edema with temporal choroidal rupture and the presence of a hematoma in the retinal domain from six o'clock to eleven o'clock. The metallic body, though penetrating deep, had not caused vascular injuries. Then proceeded to the cautious removal of the metal body after preservation of the eyeball. Successful operation, When the mydriatic drug was instilled, the pupil dilated regularly.

\section{Comments}

IOFB is an important cause of poor vision in the young population. Initial management should consist in analyzing the history of the patient (including working anamnesis) and the circumstances of trauma [1]. The ocular examination must be complete (the fundus examination is to explore the macula and papilla) and after a system-

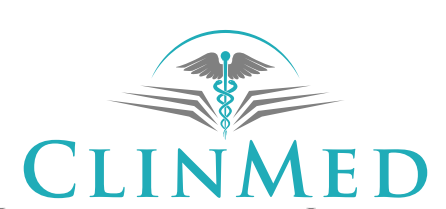

INTERNATIONAL LIBRARY

Citation: Magro VM, Magnotti MC (2017) Pre-Operative Evaluation of an Orbital Injury. Clin Med Img Lib 3:074. doi.org/10.23937/2474-3682/1510074

Received: August 26, 2017; Accepted: October 28, 2017; Published: October 30, 2017

Copyright: (c) 2017 Magro VM, et al. This is an open-access content distributed under the terms of the Creative Commons Attribution License, which permits unrestricted use, distribution, and reproduction in any medium, provided the original author and source are credited. 


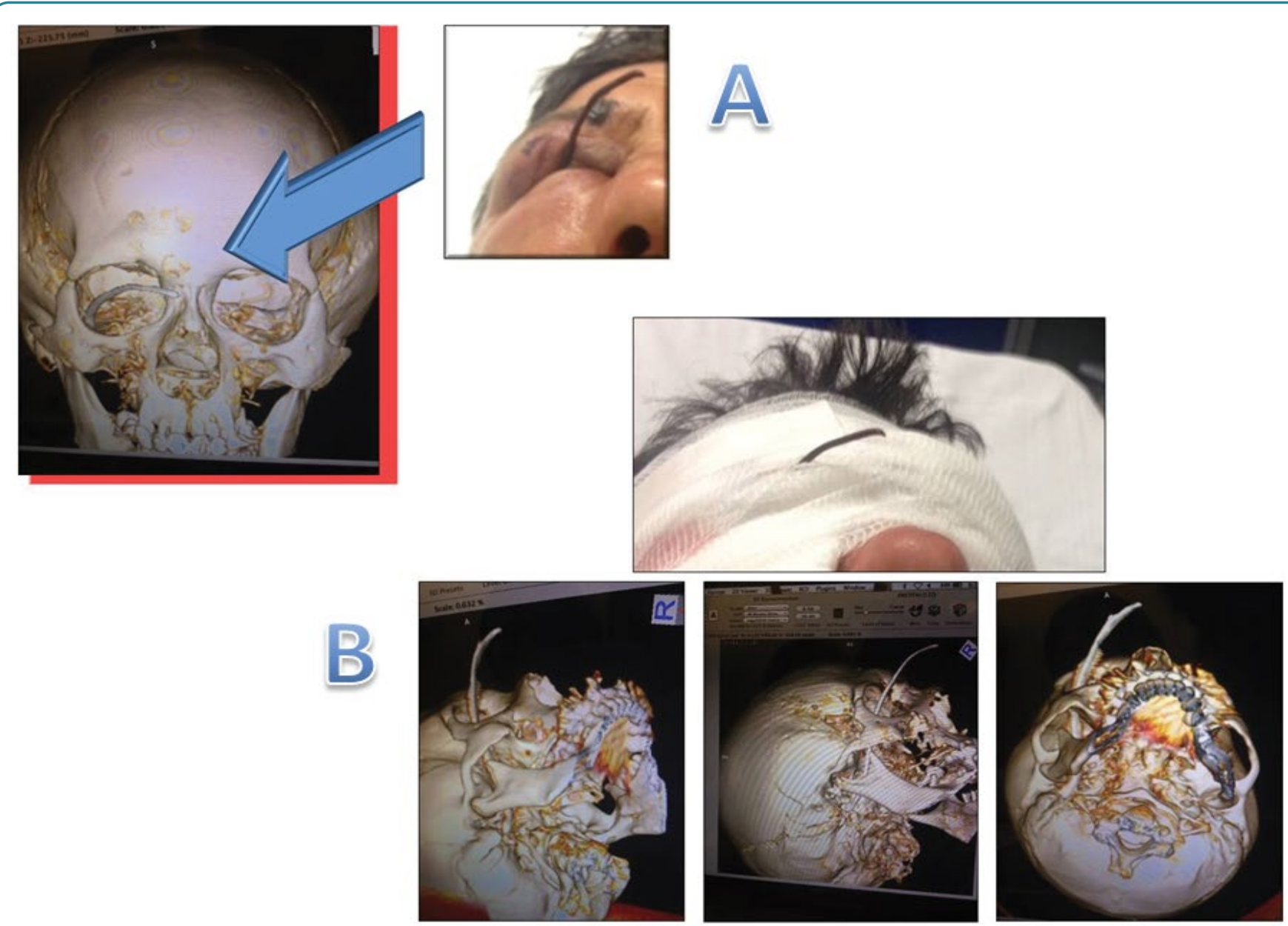

Figure 1: Panel A Three-dimensional computed tomography. We find a metal wire that protrudes outside for about $5 \mathrm{~cm}$ and penetrates into the entry point into the right orbital cavity; Panel B Three-dimensional computed tomography images showing the penetration of the metal wire inside the orbital cavity and its relationships with bones, intracranial and vascular structures.

ic antibiotic treatment has been prescribed the patient is evaluated with a CT scan. Defining surrounding bone and vascular structures by $\mathrm{CT}$ is important for surgical removal, so, for the detection of the penetrating foreign body and to evaluate the adjacent structures, we performed a three-dimensional (3D) computer-assisted detection [2,3]. The pre-operative CT scan provides a detailed analysis of the metallic intraorbital trajectory in the orbital cavity. Timely diagnosed ruptures of the posterior segment of eye before the development of traumatic cataract (late complication of (OFB), adequate therapeutic procedures prevent serious complications of IOFB penetrated eye injury such as retinal detachment and permanent reduction of visual acuity $[4,5]$. Anyway, The CT imaging findings most predictive of an open globe injury were change in globe contour and vitreous hemorrhage. Clinical exam or surgical exploration remains the most important component in evaluating for the examination of ocular globe, with $\mathrm{CT}$ imaging as an important adjunct (specially in the presence of clinically evident ocular penetration).

\section{Source of Support}

None.

\section{Conflict of Interests}

The authors state that they have no conflicts of interest.

\section{References}

1. Roman S (2001) Posterior segment intraocular foreign bodies. Initial management. J Fr Ophtalmol 24: 769-777.

2. Pokhraj PS, Jigar JP, Mehta C, Patel Narottam A (2014) Intraocular metallic foreign body: role of computed tomography. J Clin Diagn Res 8: 01-03.

3. Lin TC, Liao TC, Yuan WH, Lee FL, Chen SJ (2014) Management and clinical outcomes of intraocular foreign bodies with the aid of orbital computed tomography. J Chin Med Assoc 77: 433-436.

4. Bencić G, Vatavuk Z, Mandić Z (2004) Novel approach in the treatment of intravitreal foreign body and traumatic cataract: three case reports. Croat Med J 45: 283-286.

5. Chiquet C, Zech JC, Denis P, Adeleine P, Trepsat C (1999) Intraocular foreign bodies. Factors influencing final visual outcome. Acta Ophthalmol Scand 77: 321-325. 